\title{
miR-21 is a key therapeutic target for renal injury in a mouse model of type 2 diabetes
}

\author{
X. Zhong • A. C. K. Chung • H. Y. Chen • Y. Dong • \\ X. M. Meng • R. Li $\cdot$ W. Yang $\cdot$ F. F. Hou $\cdot$ H. Y. Lan
}

Received: 4 July 2012 / Accepted: 27 November 2012 /Published online: 5 January 2013

(C) Springer-Verlag Berlin Heidelberg 2012

\begin{abstract}
Aims/hypothesis As microRNA-21 (miR-21) plays a pathological role in fibrosis, we hypothesised that it may be a therapeutic target for diabetic nephropathy.

Methods Abundance of miR-21 was examined in diabetic kidneys from $d b / d b$ mice. The therapeutic potential of miR21 in diabetic kidney injury was examined in $d b / d b$ mice by an ultrasound-microbubble-mediated miR-21 small hairpin RNA transfer. In addition, the role and mechanisms of miR21 in diabetic renal injury were examined in vitro under
\end{abstract}

Electronic supplementary material The online version of this article (doi:10.1007/s00125-012-2804-x) contains peer-reviewed but unedited supplementary material, which is available to authorised users.

X. Zhong · A. C. K. Chung $(\bowtie) \cdot$ H. Y. Chen $\cdot$ Y. Dong $\cdot$

X. M. Meng $\cdot$ R. Li $\cdot$ H. Y. Lan

Li Ka Shing Institute of Health Sciences, The Chinese University

of Hong Kong, Prince of Wales Hospital, Shatin, The New

Territories, Hong Kong Special Administrative Region,

People's Republic of China

e-mail: chungack@cuhk.edu.hk

X. Zhong $\cdot$ R. Li

Department of Chemical Pathology, The Chinese University of Hong Kong, Shatin, Hong Kong Special Administrative

Region, People's Republic of China

\section{Zhong $\cdot$ F. F. Hou}

Division of Nephrology, Nanfang Hospital, Southern Medical

University, Research Institute of Nephrology of Guangdong

Province, Key Laboratory for Organ Failure Research, Ministry

of Education, Guangzhou, Guangdong, People's Republic of China

A. C. K. Chung $\cdot$ H. Y. Lan

Chinese University of Hong Kong Shenzhen Research Institute,

Shenzhen, People's Republic of China

H. Y. Chen $\cdot$ X. M. Meng $\cdot$ W. Yang $\cdot$ H. Y. Lan

Department of Medicine and Therapeutics, The Chinese

University of Hong Kong, Shatin, Hong Kong Special

Administrative Region, People's Republic of China diabetic conditions in rat mesangial and tubular epithelial cell lines by overexpressing or downregulating miR-21.

Results In $d b / d b$ mice, a mouse model of type 2 diabetes, renal miR-21 at age 20 weeks was increased twofold compared with $\mathrm{db} / \mathrm{m}^{+}$mice at the same age, and this increase was associated with the development of microalbuminuria and renal fibrosis and inflammation. More importantly, gene transfer of miR-21 knockdown plasmids into the diabetic kidneys of $d b / d b$ mice at age 10 weeks significantly ameliorated microalbuminuria and renal fibrosis and inflammation at age 20 weeks, revealing a therapeutic potential for diabetic nephropathy by targeting miR-21. Overexpression of miR-21 in kidney cells enhanced, but knockdown of miR-21 suppressed, high-glucose-induced production of fibrotic and inflammatory markers. Targeting Smad7 may be a mechanism by which miR-21 regulates renal injury because knockdown of renal miR-21 restored Smad7 levels and suppressed activation of the TGF- $\beta$ and NF- $\mathrm{KB}$ signalling pathways.

Conclusions/interpretation Inhibition of miR-21 might be an effective therapy for diabetic nephropathy.

Keywords Diabetic nephropathy $\cdot$ MicroRNA-21 - TGF- $\beta$ signalling $\cdot$ Smad 7

\begin{tabular}{ll}
\multicolumn{2}{l}{ Abbreviations } \\
DN & Diabetic nephropathy \\
Dox & Doxycycline \\
MC & Mesangial cell \\
MCP & Monocyte chemotactic protein \\
miR-21 & MicroRNA-21 \\
miR-21 KD & miR-21 knockdown \\
NF-kB & Nuclear factor $\kappa B$ \\
shRNA & Small hairpin RNA \\
siRNA & Small interfering RNA \\
T $\beta$ RII & TGF- $\beta$ receptor II \\
TEC & Tubular epithelial cell \\
UTR & Untranslated region
\end{tabular}




\section{Introduction}

Diabetes is a chronic metabolic syndrome due to insulin deficiency, and its vascular complications are responsible for morbidity and mortality in diabetic patients. Diabetic nephropathy (DN) is a major complication of diabetes that leads to end stage renal disease. Among numerous mediators of DN $[1,2]$, TGF- $\beta 1$ is the key one [3]. In vitro studies have shown that induction of extracellular matrix production by high glucose and AGE is TGF- $\beta$-dependent [3]. Results from animal models of both type 1 and type 2 diabetes confirm that TGF- $\beta$ is an essential mediator of diabetic kidney injury [3]. Targeting TGF- $\beta$ signalling should have therapeutic potential against DN. However, blockade of TGF- $\beta$ signalling may induce inflammation, as $T g f b 1$ knockout mice die from massive inflammation [4]. Alternative approaches to inhibiting TGF- $\beta$ action during DN should be developed in an attempt to protect against renal injury.

Recently, numerous studies have demonstrated that TGF- $\beta$ regulates specific microRNAs, influencing renal fibrosis in kidney diseases [5-9]. MicroRNA-21 (miR-21) is the most significant microRNA involved in fibrotic disorders, and its levels are upregulated in human kidney diseases $[5,10,11]$. In a previous study, we demonstrated that TGF- $\beta 1$ increases miR-21 content during renal fibrosis via a Smad3-dependent mechanism [7]. Suppression of miR-21 abundance in a mouse model of obstructive kidney disease ameliorates renal fibrosis and halts the progression of renal fibrosis in an established obstructive nephropathy $[7,12]$, revealing a therapeutic potential for miR-21 knockdown in kidney diseases.

Thus, in this study, we hypothesised that targeting miR21 may have therapeutic potential for DN. First, we determined whether knockdown of miR-21 in vivo would halt the progression of renal injury in $d b / d b$ mice. We determined the role of miR-21 in high-glucose-induced renal fibrosis and inflammation by altering miR-21 levels in kidney cells. Finally, we determined whether $\operatorname{Smad} 7$ was a target of miR-21 during DN.

\section{Methods}

Cell culture The normal rat mesangial cell (MC) line, 1099, and tubular epithelial cell (TEC) line, NRK52E (ATCC, Manassas, VA ,USA) were maintained in DMEM/low glucose $(5.5 \mathrm{mmol} / \mathrm{l}$; Life Technologies, Grand Island, NY, USA) containing 5\% FBS and $0.1 \%$ antibiotic/antimycotic solution (Life Technologies), in six-well plastic plates at $37^{\circ} \mathrm{C}$ in an incubator with $5 \% \mathrm{CO}_{2}$. Cells were stimulated with D-glucose at normal $(5.5 \mathrm{mmol} / \mathrm{l})$ or high (25 mmol/l) concentration for periods of $0,24,48$ and $72 \mathrm{~h}$ in serum-free medium, in accordance with our own published procedures [13]. D-Mannitol $(25 \mathrm{mmol} / \mathrm{l}$; Life Technologies) was used as a control for osmolality.

To impair TGF- $\beta$ signalling, a stable dominant negative form of the TGF- $\beta$ receptor II (T $\beta$ RII)-producing NRK-52E cell line was used as previously described [14].

Construction of inducible cell lines of doxycycline (Dox)-regulated miR-21 overexpression and knockdown was performed as reported previously [7]. At $48 \mathrm{~h}$ before the treatment, Dox $(0.5 \mu \mathrm{g} / \mathrm{ml})$ was added to induce the levels of miR-21 or miR-21 small hairpin RNA (shRNA). The cells were then stimulated with DMEM/high glucose $(25 \mathrm{mmol} / \mathrm{l}$; Life Technologies) for $48 \mathrm{~h}$ in serumfree medium, in accordance with our own published procedures [13].

Transient transfection MCs and TECs were transfected with $30 \mathrm{nmol} / 1$ Silencer-select Smad7 small interfering RNA (siRNA) or a negative control of siRNA (Life Technologies) in six-well plates using siPort Neo-FX (Life Technologies) according to the manufacturer's instructions [8]. After transient transfection, we synchronised the cells by culturing them in DMEM/low glucose medium without serum for $24 \mathrm{~h}$. The cells were then stimulated with DMEM/high glucose medium for $48 \mathrm{~h}$ following our protocols [13].

Dox-regulated miR-21-overexpressing TECs were transfected with 1, 2 and $4 \mu \mathrm{g} /$ well of pcDNA3.1-Smad 7 expression plasmid or empty pcDNA 3.1 plasmid (Life Technologies) in six-well plates using Lipofectamine LTX (Life Technologies) according to the manufacturer's instruction. At $48 \mathrm{~h}$ before the treatment, Dox $(0.5 \mu \mathrm{g} / \mathrm{ml})$ was added to increase the abundance of miR-21. After transient transfection, we synchronised the cells by culturing them in DMEM/low glucose medium without serum for $24 \mathrm{~h}$. The cells were then stimulated with DMEM/high glucose medium for $48 \mathrm{~h}$ following our protocols [13].

Animal model Adult, male BKS.Cg-Dock $7^{m}+/+L e p r^{d b} / \mathrm{J}$ $d b / d b$ mice with a C57BLKS/J background (Jackson Laboratory, Bar Harbor, ME, USA), at 10 weeks of age, denoting the overt phases of the type 2 diabetes syndrome [13], were used in these studies. Details of animal models are provided in electronic supplementary material (ESM) Methods. All animal studies were approved by the Chinese University of Hong Kong's Animal Experimental Ethics Committee.

Ultrasound-mediated gene transfer of inducible miR-21 knockdown (miR-21 KD) plasmids into the kidneys of db/ $d b$ mice The mixture of miR-21 KD and pDNA6/TR tet-on plasmids was combined with Sonovue (Bracco, Milan, Italy) at a ratio of 1:1 (vol./vol.) as described previously $[7,9,15-18]$. The mixed solution $(400 \mu \mathrm{l})$ was then injected via the tail vein of eight $d b / d b$ mice (20-22 g body weight, 
6 weeks old). The ultrasound transducer (Therasonic, ElectroMedical Supplies, Wantage, England) was then applied to the kidneys using ultrasound medium with a continuous wave output of $1 \mathrm{MHz}$ at $1 \mathrm{~W}$ power output for a total of $5 \mathrm{~min}$ for each side. The same procedure was applied to eight control animals with a mixture containing the same amount of empty control plasmids (pSuperior/pcDNA6). To induce transgene expression at the optimal dosage, a dose of Dox solution $(200 \mu \mathrm{g} / \mathrm{ml}$ in $200 \mu \mathrm{l}$ volume) was first injected into the peritoneal cavity after the gene transfer. The mice were then given drinking water containing Dox $(200 \mu \mathrm{g} / \mathrm{ml})$ until they were killed [16]. To maintain the transgene expression levels, gene therapy was given at age 10,13 and 16 weeks. A group of eight age-matched $\mathrm{db} / \mathrm{m}$ mice was used as normal controls. All mice were killed at age 20 weeks, and their kidneys were collected for analysis. The experimental procedures were approved by the Chinese University of Hong Kong's Animal Experimental Ethics Committee.

In situ hybridisation A specific 5'-FITC-labelled antisenselocked nucleic acid oligonucleotide for mmu-miR-21 and a scramble probe as a negative control were purchased from Exiqon (Vedbaek, Denmark). The detailed procedure for in situ hybridisation was carried out according to the manufacturer's protocol [19]. In brief, 10-14 $\mu \mathrm{m}$ slides were prepared from optimal cutting temperature compoundembedded kidney tissues. The slides were then fixed in $4 \%$ paraformaldehyde for $10 \mathrm{~min}$, followed by prehybridisation with $1 \times$ hybridisation buffer (Exiqon) without the probe. The hybridisation was carried out overnight in $1 \times$ hybridisation buffer (30-70 $\mu \mathrm{l})$ with FITC/anti-sense microRNA probe at $45^{\circ} \mathrm{C}$ overnight. After being washed, the slides were blocked and incubated with Smad7 antibody (Abcam, Cambridge, MA, USA), and subsequently with Alexa Fluor 555-conjugated secondary antibody (Life Technologies, Carlsbad, CA, USA). Signals were visualised and detected under a fluorescence microscope (Axioplan2 Imaging; Carl Zeiss, Oberkoche, Germany).

$R N A$ extraction and quantitative RT-PCR analyses, western blot analysis, urine albumin excretion measurement, histology, immunohistochemistry and luciferase reporter assay Details of these methods are provided in ESM Methods.

Construction of Smad7 overexpression plasmid and plasmids for luciferase reporter assay Details of the cloning procedure are provided in ESM Methods.

Statistical analysis Data from real-time PCR and immunohistochemical and western blot analysis were expressed as mean \pm SEM and compared using ANOVA with the Newman-Keuls comparison program from GraphPad Prism 5.0 (GraphPad Software, San Diego, CA, USA).

\section{Results}

Knockdown of miR-21 in db/db mice reduces miR-21 levels, improves renal function, and inhibits renal fibrosis and inflammation during kidney damage caused by type 2 diabetes We found that at the age of 10 weeks, renal miR-21 content in $d b / d b$ mice was already significantly higher than in the $d b /$ $\mathrm{m}^{+}$littermates (Fig. 1a). At age 20 weeks, renal miR-21 was twofold higher in $d b / d b$ mice. This result provides the first in vivo evidence that miR-21 levels may play a role in DN.

We then investigated whether knockdown of miR-21 prevented renal injury in $d b / d b$ mice by delivering miR-21 KD plasmids into kidneys with ultrasound-microbubble-mediated gene transfer at age 10,13 and 16 weeks (Fig. 1b). As described previously [7], this knockdown plasmid targets the stem loop sequence of pre-miR-21 and suppresses renal miR21 abundance for at least 14 days [7]. The dose of miR-21 shRNA was carefully increased by Dox to avoid inducing dramatic cell death in diabetic kidneys [7].

At age 20 weeks, $d b / d b$ mice developed similar levels of hyperglycaemia regardless of the treatment (blood glucose concentration $30.15 \pm 0.78 \mathrm{mmol} / \mathrm{l}$ with control plasmids and $30.97 \pm 1.15 \mathrm{mmol} / 1$ with miR-21 KD plasmids), indicating that delivery of miR-21 KD plasmids did not affect blood glucose levels. In addition, $d b / d b$ mice treated with miR$21 \mathrm{KD}$ plasmids developed less severe microalbuminuria than normal $d b / d b$ mice over the 20-week disease course (Fig. 1c). Pathologically, an increase in mesangial matrix and thickness of the glomerular basement membrane was apparent in $d b / d b$ mice at week 20 after diabetes onset, which was reduced in diabetic mice receiving miR-21 shRNA treatment (Fig. 1d, e). These results suggest that in vivo inhibition of miR-21 improved renal function in type 2 diabetic mice. Compared with $d b / m^{+}$littermates, the renal content of $T G F-\beta 1$ and miR-21 in $d b / d b$ mice was elevated from the age of 10 weeks (Fig. 1f, g). However, delivery of miR-21 KD plasmids into the diabetic kidneys of $d b / d b$ mice suppressed the renal content of $T G F-\beta 1$ and miR-21 at age 20 weeks to the levels of 10 -week-old $d b / d b$ mice, suggesting that progression of diabetic kidney injury was suppressed (Fig. 1f, g). No effect was observed when control plasmids with scrambled RNA sequence [7] were used (Fig. 1f, g).

Immunohistochemistry revealed that, although increased collagen I deposition in diabetic kidneys was largely confined to the area of tubulointerstitium, and abundant collagen IV accumulation was noted in both glomerular and tubulointerstitial areas, their levels were largely reduced in $d b / d b$ mice treated with miR-21 shRNA compared with mice treated with control plasmids (Fig. 2a-d). Real-time PCR and western blot analyses also demonstrated that, compared with the 10-week-old $d b / d b$ mice, there was a $20-30 \%$ increase in both mRNA and protein abundance of 

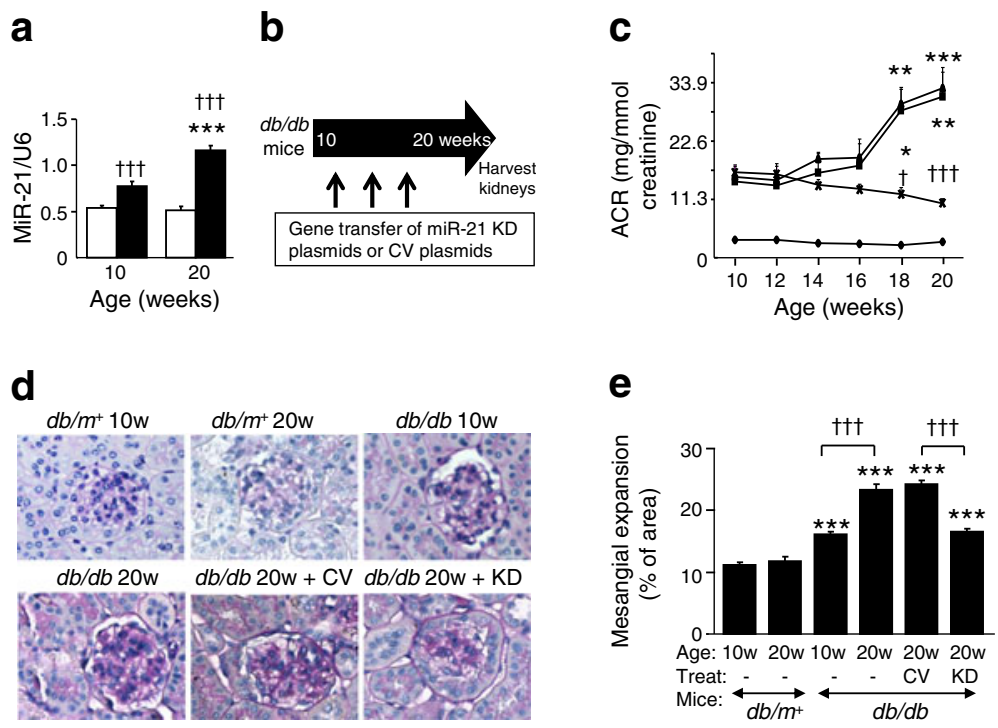

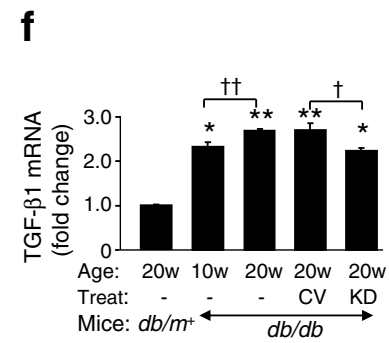

Fig. 1 Delivery of miR-21 KD plasmids reduces renal abundance of TGF- $\beta 1$ and miR-21 and improves renal function in 20-week-old $d b$ / $d b$ mice. (a) Renal miR-21 content in $d b / d b$ mice (black bars) and $d b /$ $\mathrm{m}^{+}$littermates (white bars) at age 10 and 20 weeks. (b) Schematic diagram of the experimental approach. (c) Urinary albumin to creatinine ratio (ACR). Circles, $d b / m^{+}$; squares, $d b / d b$; triangles, $d b / d b+$ $\mathrm{CV}$; crosses, $d b / d b+\mathrm{KD}$. (d) Histology (periodic acid-Schiff [PAS]stained sections). (e) Mesangial expansion. (f) Renal TGF- $\beta 1$ mRNA level. (g) Renal miR-21 abundance. Real-time PCR results show that renal TGF- $\beta 1$ and miR-21 content in diabetic kidneys are increased at g

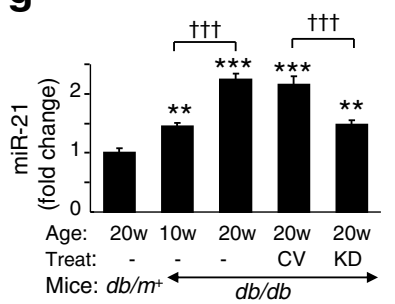

age 10 and 20 weeks, but are decreased after gene transfer of miR$21 \mathrm{KD}$ plasmids. $d b / d b$ mice with miR-21 KD treatment develop less severe microalbuminuria and less mesangial expansion than the normal $d b / d b$ mice over the 20 -week disease course. Levels of miR21 are normalised to U6 small nuclear RNA (miR-21/U6). Each bar represents the mean \pm SEM for at least eight mice. ${ }^{*} p<0.05$, ${ }^{* *} p<0.01$, ${ }^{* * *} p<0.001$ compared with $\mathrm{db} / \mathrm{m}^{+}$mice; ${ }^{\dagger} p<0.05,{ }^{\dagger \dagger} p<0.01,{ }^{\dagger \dagger} p<0.001$ as indicated or compared with $d b / m^{+}$mice or $d b / d b+C V$ at the same time point. CV, control vectors; KD, miR-21 KD plasmids; w, weeks. Magnification: $\times 400$ fibrotic markers at 20 weeks (Fig. 3a-g), whereas treatment with miR-21 KD plasmids reduced abundance of these markers to the levels at 10 weeks (Fig. 3a-g). These findings suggest that the reduction in miR-21 levels halted the progression of renal fibrosis, as evidenced by the decrease in synthesis and deposition of extracellular matrix.

Owing to the essential role of inflammation during DN $[20,21]$, we also examined whether suppression of miR-21 influenced renal inflammation under diabetic conditions. Quantification of immunohistochemical results showed that the amount of macrophage infiltration into diabetic kidneys was increased after age 10 weeks, but this increase was markedly reduced after treatment with miR-21 KD plasmids (Fig. 4a, b). Similarly, the proinflammatory cytokine TNF$\alpha$, and the chemokine monocyte chemotactic protein (MCP)-1, were upregulated in diabetic kidneys of $d b / d b$ mice after 10 weeks of age compared with their $\mathrm{db} / \mathrm{m}^{+}$ littermates. Suppression of miR-21 caused a reduction in both TNF- $\alpha$ and MCP-1 in diabetic kidneys (Fig. 4a, c and d; ESM Fig. 1a, b), implying that miR-21 plays a role in renal inflammation during $\mathrm{DN}$.

MiR-21 is an important mediator of high-glucose-induced renal fibrosis and inflammation in vitro To confirm the role of miR-21 in kidneys under diabetic conditions, we first determined whether a high-glucose concentration upregulated miR-21 abundance in both MCs and TECs. Highglucose concentration, but not the controls, induced miR21 abundance in a time-dependent manner in both MCs and TECs (ESM Fig. 2a). This upregulation was blocked by the presence of the dominant negative form of T $\beta$ RII (ESM Fig. $2 \mathrm{~b}$ ), implying that TGF- $\beta$ signalling was necessary for the high-glucose-induced miR-21 level. 
Fig. 2 Delivery of miR-21 KD plasmids inhibits renal abundance of collagen I and IV in 20-week-old $d b / d b$ mice. (a) Immunohistochemistry of collagen I (COL I) and IV (COL IV). Quantitative analysis of immunohistochemical staining of (b) COL I, (c) glomerular COL IV, and (d) interstitial COL IV. Results show that when compared with diabetic mice $(d b / d b)$ and diabetic mice with control vector (CV) treatment, miR-21 KD gene transfer significantly inhibits renal collagen I and IV production. Each bar represents the mean \pm SEM for at least eight mice. ${ }^{*} p<0.05$, $* * * p<0.001$ compared with $\mathrm{db} / \mathrm{m}^{+}$mice at age 10 weeks; ${ }^{\dagger \dagger} p<0.001$ as indicated. KD, miR-21 KD plasmids; w, weeks. Magnification: $\times 400$ a

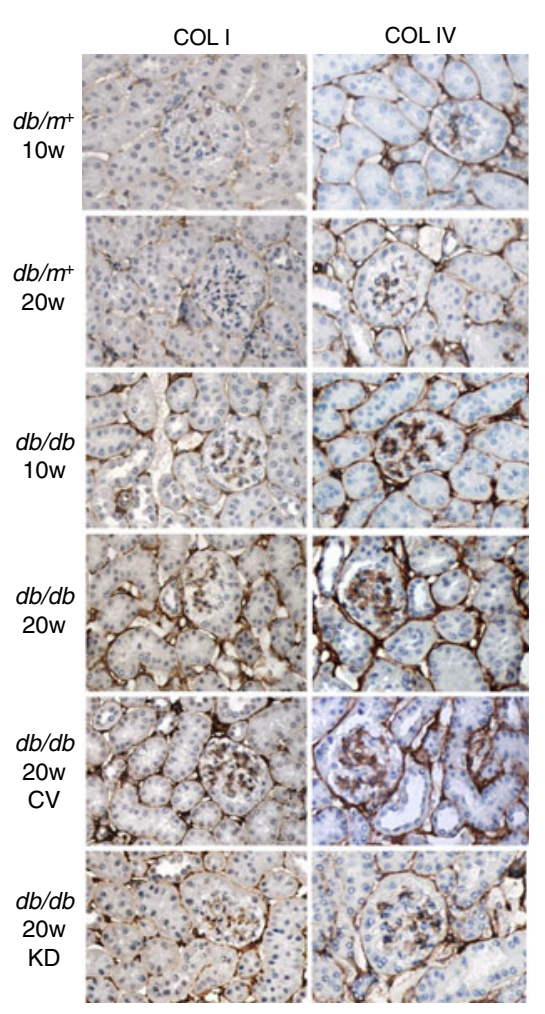

b
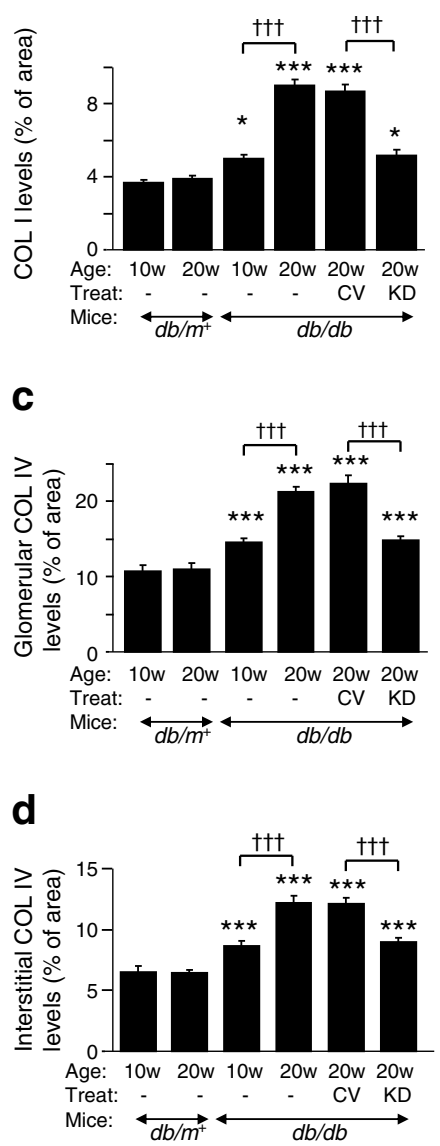

We next either overexpressed miR-21 (Fig. 5a, e) or inhibited miR-21 (Fig. 5i, m) in MCs and TECs to study the functional role of miR-21 in high-glucose-induced renal fibrosis and inflammation. Overexpression of miR-21 further magnified the high-glucose-induced abundance of fibrotic markers, such as collagen I, collagen IV and fibronectin, in MCs and TECs (MCs, Fig. 5b-d and ESM Fig. 2c, d; TECs, Fig. 5f-h and ESM Fig. 2e, f), but knockdown of miR-21 in cells significantly attenuated the abundance of these markers (MCs, Fig. 5j-1 and ESM Fig. 2g, h; TECs, Fig. 5n-p and ESM Fig. 2i, j). Similarly, miR-21 overexpression further promoted increases in the levels of proinflammatory markers (Tnf- $\alpha, I l-1 \beta, M c p$ 1 and intercellular adhesion molecule (Icam)-1) (Fig. 6a-d and ESM Fig. 1c, d) under diabetic conditions, which was reversed by knocking down the miR-21 levels (Fig. 6e$\mathrm{h}$ and ESM Fig. 1e, f). These results provide strong evidence that miR-21 plays a pathological role in highglucose-mediated renal fibrosis and inflammation and support the therapeutic effect of targeting miR-21 to block the progression of renal fibrosis and inflammation by delivering miR-21 KD plasmids into diabetic kidneys.
Smad7 is a target of miR-21 during renal fibrosis and inflammation The next question to ask was how miR-21 affects both renal fibrosis and inflammation. Therefore, we examined the signalling pathways related to renal fibrosis and inflammation, such as the TGF- $\beta$ and NF- $k B$ pathways, in diabetic kidneys. After 10 weeks of age, TGF- $\beta 1$ protein was upregulated in kidneys from $d b / d b$ mice (Fig. 7a). At the same time, both phosphorylated Smad3 and NF-kB-p65 were increased, implying that both TGF- $\beta$ and NF- $k B$ pathways were activated during diabetic renal injury. After treatment with miR- $21 \mathrm{KD}$ plasmids, TGF- $\beta 1$ protein, phosphorylated Smad3 and NF- $\mathrm{kB}-\mathrm{p} 65$ were observed to decline (Fig. 7a), suggesting that suppression of miR-21 inhibited activation of the TGF- $\beta$ and NF- $\mathrm{KB}$ signalling pathways.

As reported in lung fibrosis, Smad 7 is a target of miR-21 [22], and overexpression of Smad7 can suppress the TGF- $\beta$ and NF- $\mathrm{KB}$ pathways $[16,23]$. We therefore examined whether renal Smad7 abundance was also altered after miR-21 inhibition. Consistent with the type 1 diabetic mouse model [24], Smad7 (both RNA and protein levels) was significantly reduced during diabetic kidney injury in 
Fig. 3 Delivery of miR-21 KD plasmids slows the progression of renal fibrosis in $d b / d b$ mice. (a) Representative western blots; (b-d) quantitative analysis of western blots; (e-g) Realtime PCR analysis. Levels of Col I (also known as Collal), $\mathrm{Fn} 1$ and $\mathrm{Col} I V$ (also known as Col4al) in diabetic kidneys are increased at age 10 and 20 weeks but decreased after gene transfer of miR-21 KD plasmids. Each bar represents the mean \pm SEM for at least eight mice.

${ }^{*} p<0.05, * * p<0.01, * * * p<$ 0.001 compared with $\mathrm{db} / \mathrm{m}^{+}$ mice; ${ }^{\dagger \dagger} p<0.01,{ }^{\dagger \dagger} p<0.001$ as indicated. $\mathrm{CV}$, control vectors; $\mathrm{KD}$, miR-21 KD plasmids; w, weeks
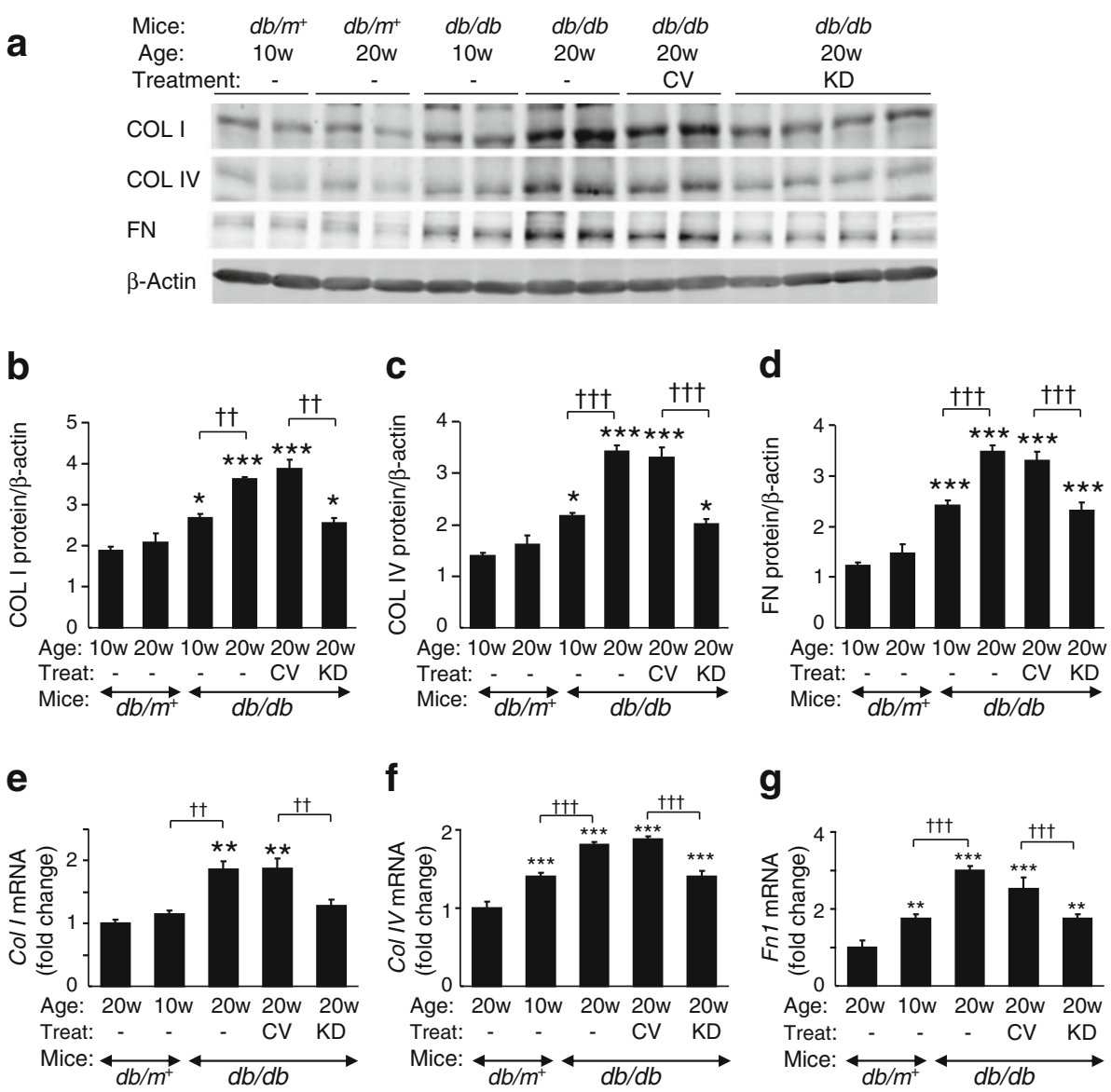

f

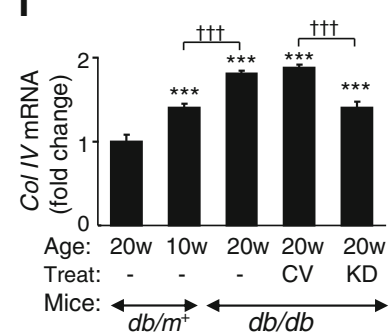

g

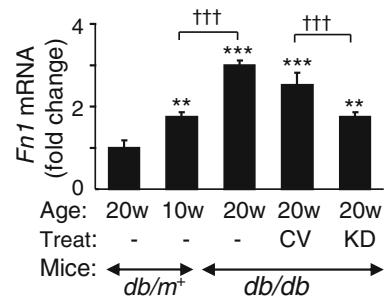

$d b / d b$ mice aged 20 weeks (Fig. 7a and ESM Fig. 3a, b). Suppression of miR-21 partially restored the Smad7 content of 20 -week-old $d b / d b$ mice to that at age 10 weeks. Results from in situ hybridisation with FITC-miR-21 showed that miR-21 was induced in both glomeruli and renal tubules during DN (Fig. 7b). More significantly, results from a combination of immunohistochemical staining with Smad7 antibody and in situ hybridisation with FITC-miR-21 showed that the levels of renal Smad7 correlated negatively with miR-21 abundance during renal fibrosis (Fig. 7b). This negative correlation was confirmed by the results from in vitro studies. Overexpression of miR-21 further decreased the Smad7 mRNA content in both MCs and TECs, while knockdown of miR-21 restored Smad7 levels (ESM Fig. 3c-f).

To assess the functional contributions of loss of $\operatorname{Smad} 7$ to renal fibrosis and inflammation, we then examined whether knocking down Smad7 promoted the highglucose-induced renal fibrosis and inflammation, by transiently transfecting Smad7 siRNA into MCs and TECs. Expression of collagen I, fibronectin, Tnf- $\alpha$ and $I l-1 \beta$ induced by high glucose was significantly enhanced by treatment with Smad7 siRNA (Fig. 8a-f and ESM Fig. 3g-j), confirming that $\mathrm{Smad} 7$ plays a protective role in renal fibrosis and inflammation.
To determine whether Smad7 acted downstream of miR21 function, we overexpressed Smad7 in Dox-induced miR21-overexpressing TECs by transiently transfecting different dosages of Smad7 overexpression plasmid without the $3^{\prime}$ untranslated region (UTR) into Dox-induced miR-21expressing TECs. Results of real-time PCR and western blot assays demonstrated that miR-21 overexpression increased the high-glucose-induced mRNA content of collagen I, fibronectin, Tnf- $\alpha$ and $I l-1 \beta$ (Fig. 8g, h and ESM Fig. 4a-e). Overexpression of $\mathrm{Smad} 7$ resulted in $>1.5$-fold increase in abundance of Smad7, thereby largely reversing the stimulating effect of miR-21 on high-glucose-induced expression of collagen I, fibronectin, $T n f-\alpha$ and $I l-1 \beta$ in a dosedependent manner (Fig. 8g, h and ESM Fig. 4a-e). Thus, overexpression of Smad7 in miR-21-overexpressing renal cells reduced the abundance of inflammatory and fibrosis markers, suggesting that Smad7 is downstream of miR-21.

To determine whether miR-21 directly regulates Smad7 content, we assessed the complementarity of miR-21 to the Smad7 3'-UTR using TargetScan (http://www.targetscan.org/; accessed 7 February 2012). The computational algorithm from TargetScan predicted miR-21-binding sites in the Smad7 3 '-UTR that are highly conserved between humans and rodents (ESM Fig. 4f), suggesting that Smad7 may be a 
Fig. 4 Delivery of miR-21 KD plasmids slows the progression of renal inflammation in $d b / d b$ mice. (a) Immunohistochemistry. (b-d) Quantitative analysis of immunohistochemical staining of F4/80-positive cells and content of TNF- $\alpha$ and MCP-1, respectively. F4/80-positive cells and abundance of TNF- $\alpha$ and MCP-1 in diabetic kidneys are increased at age 10 and 20 weeks, but decreased after gene transfer of miR-21 KD plasmids. Each bar represents the mean \pm SEM for at least eight mice. ${ }^{*} p<0.05,{ }^{* *} p<$ $0.01, * * * p<0.001$ compared with $\mathrm{db} / \mathrm{m}^{+}$mice at 10 weeks; ${ }^{\dagger} p<0.05,{ }^{\dagger \dagger} p<0.001$ as indicated. $\mathrm{CV}$, control vectors; $\mathrm{KD}$, miR-21 KD plasmids; $w$, weeks. Magnification: $\times 400$ for F4/80 staining and $\times 200$ for TNF- $\alpha$ and MCP- 1 staining a

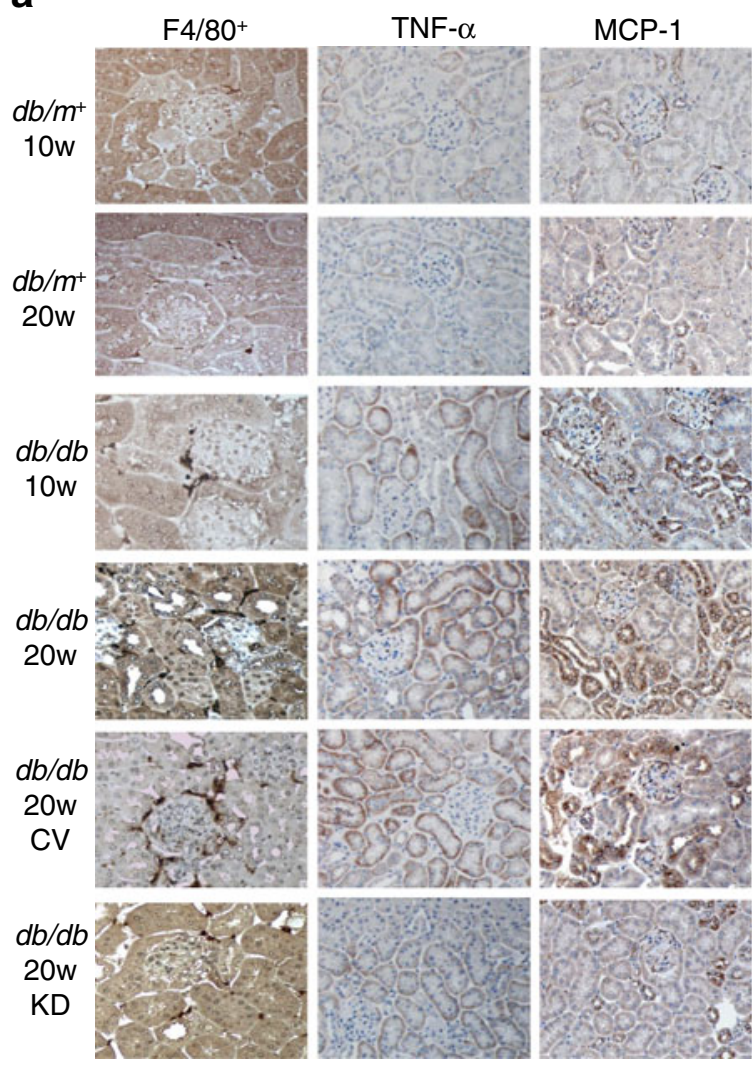

b

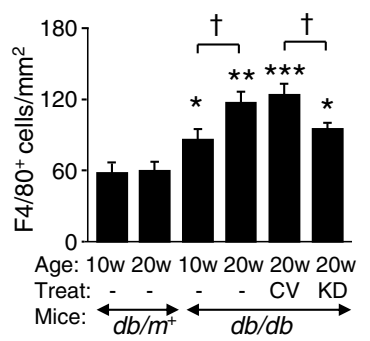

C

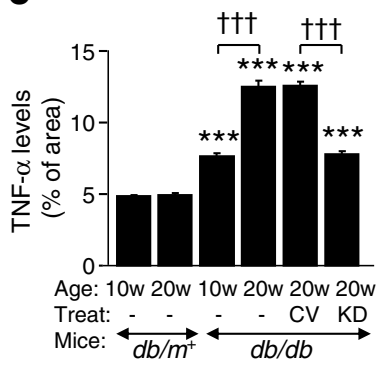

d

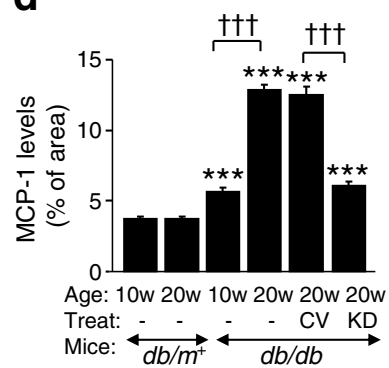

miR-21 target. Therefore we subcloned various fragments of mouse Smad7 3'-UTR and sequences with native and mutated miR-21-binding sites downstream of the luciferase gene. Overexpression of miR-21 significantly downregulated luciferase activity in the reporter constructs containing the entire Smad7 3'-UTR or the 3'-UTR with a miR-21-binding site compared with normal TECs (Fig. 8i). Furthermore, miR-21 overexpression significantly reduced luciferase activity in the reporter containing the sequences with a native, but not mutant, miR-21binding site. Collectively, these results suggest that miR21 binds to the Smad7 3'-UTR and downregulates Smad7 abundance to derepress $\mathrm{TGF}-\beta$ and $\mathrm{NF}-\mathrm{KB}$ signalling pathways, which are enhanced in renal fibrosis and inflammation during DN.

\section{Discussion}

Our findings identify miR-21 as an important signature microRNA in DN because renal miR-21 was shown to be markedly upregulated in diabetic kidneys of $d b / d b$ mice, and this is associated with the development of microalbuminuria and renal fibrosis and inflammation. More importantly, our study shows that in vivo inhibition of miR-21 ameliorated progression of DN in an established experimental model of type 2 diabetes. Suppressing $\operatorname{Smad} 7$ levels to promote activation of the TGF- $\beta$ and NF- $\mathrm{KB}$ signalling pathways may be the mechanism whereby miR-21 promotes renal fibrosis and inflammation in diabetic kidney injury. Targeting miR-21 may be a good approach to preventing diabetic kidney injury. 
a

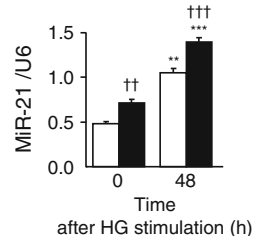

e

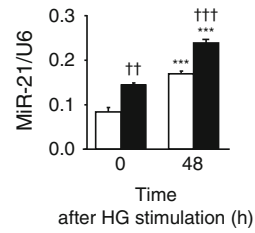

i

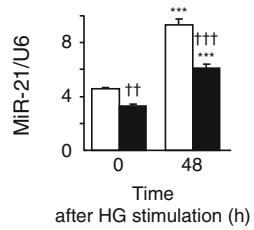

m

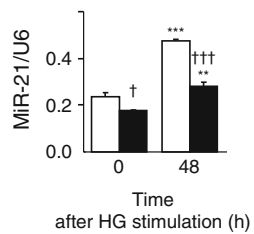

b

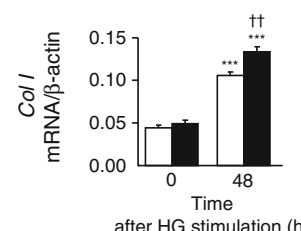

f

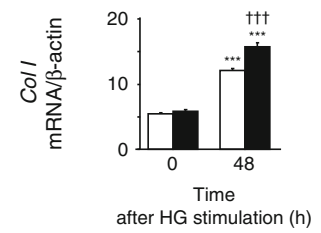

j

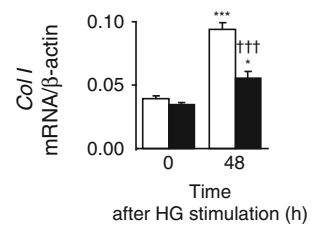

n

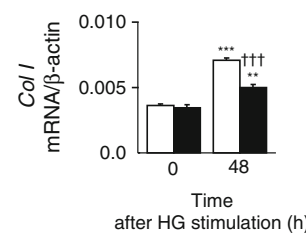

C

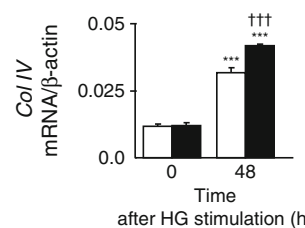

g

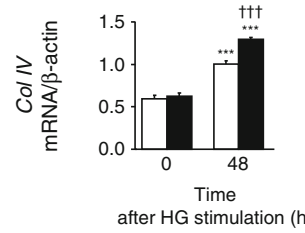

k

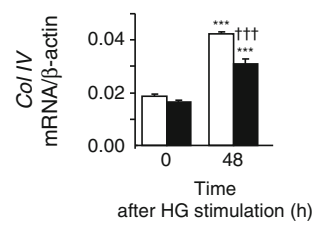

0

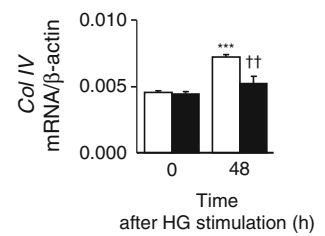

d

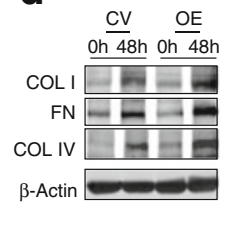

h

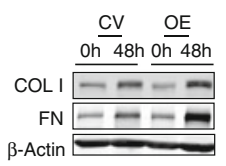

I

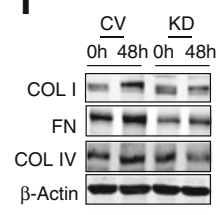

p

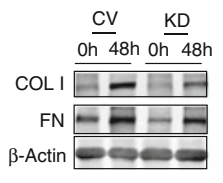

Fig. 5 Alteration of miR-21 level affects high-glucose (HG)-induced abundance of fibrotic markers in rat MCs and TECs. (a) Real-time PCR results of miR-21 in MCs with overexpression of miR-21. (b, c) Real-time PCR and (d) representative western blot results of fibrotic markers in MCs with overexpression of miR-21. (e) Real-time PCR results of miR-21 in TECs with overexpression of miR-21. (f, g) Realtime PCR and (h) representative western blot results of fibrotic markers in TECs with overexpression of miR-21. White bars, + control vectors (CV); black bars, + miR-21 overexpression plasmids (OE). (i) Realtime PCR results of miR-21 in MCs with knockdown of miR-21. (j, k) Real-time PCR and (l) representative western blot results of fibrotic markers in MCs with knockdown of miR-21. (m) Real-time PCR

We have previously shown that, during renal fibrosis, TGF$\beta 1$ upregulates the miR-21 level via a Smad3-dependent mechanism [7]. Results from this study confirm that TGF- $\beta$ signalling is necessary for high-glucose-induced miR-21 production. Upregulation of renal miR-21 content during $\mathrm{DN}$ in mouse models of type 1 [25] and type 2 diabetes shows a positive correlation between miR-21 and progression of renal injury in DN. This study further shows that, under diabetic conditions, overexpression of miR-21 increases renal fibrosis, but suppression of miR-21 reduces renal fibrosis. These results are consistent with the pathological role of miR-21 in cardiac, lung and renal fibrosis [7, 22, 26]. Furthermore, we demonstrate for the first time that overexpression of miR-21 in

results of miR-21 in TECs with knockdown of miR-21. (n, o) Realtime PCR and (p) representative western blot results of fibrotic markers in TECs with knockdown of miR-21. White bars, + CV; black bars, + miR-21 KD plasmids. Overexpression of miR-21 in MCs and TECs increases levels of collagen I (COL I), fibronectin (FN) and collagen IV (COL IV) induced by high glucose for $48 \mathrm{~h}$, while knockdown of miR-21 in MCs and TECs inhibits increases in levels of COL I, FN and COL IV. Levels of miR-21 are normalised to U6 small nuclear RNA (miR-21/U6). Each bar represents the mean \pm SEM for at least three independent experiments. ${ }^{*} p<0.05,{ }^{* *} p<0.01,{ }^{* * *} p<0.001$ compared with time $0 ;{ }^{\dagger} p<0.05,{ }^{\dagger \dagger} p<0.01,{ }^{\dagger \dagger} p<0.001$ compared with $\mathrm{CV}$ at the same time point

kidney cells promotes, and knockdown of miR-21 reduces, renal inflammation under diabetic conditions. Thus, inhibiting both renal fibrosis and inflammation by in vivo suppression of miR-21 may be the mechanism by which miR-21 ameliorates progression of $\mathrm{DN}$ in type 2 diabetes.

In this study, we identified $\operatorname{Smad} 7$ as a target of miR-21 during renal injury and demonstrated that overexpression of Smad7 was able to reverse the pathological effects of miR21 on renal fibrosis and inflammation. These findings are significant because, during DN, renal abundance of Smad7 is downregulated and gene therapy with Smad7 ameliorates proteinuria and suppresses both renal fibrosis and inflammation [24]. Overexpression of Smad7 can suppress the 


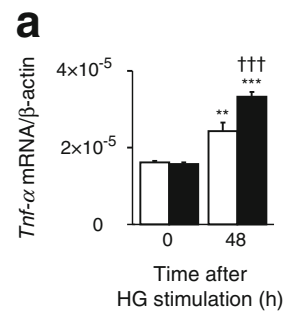

b
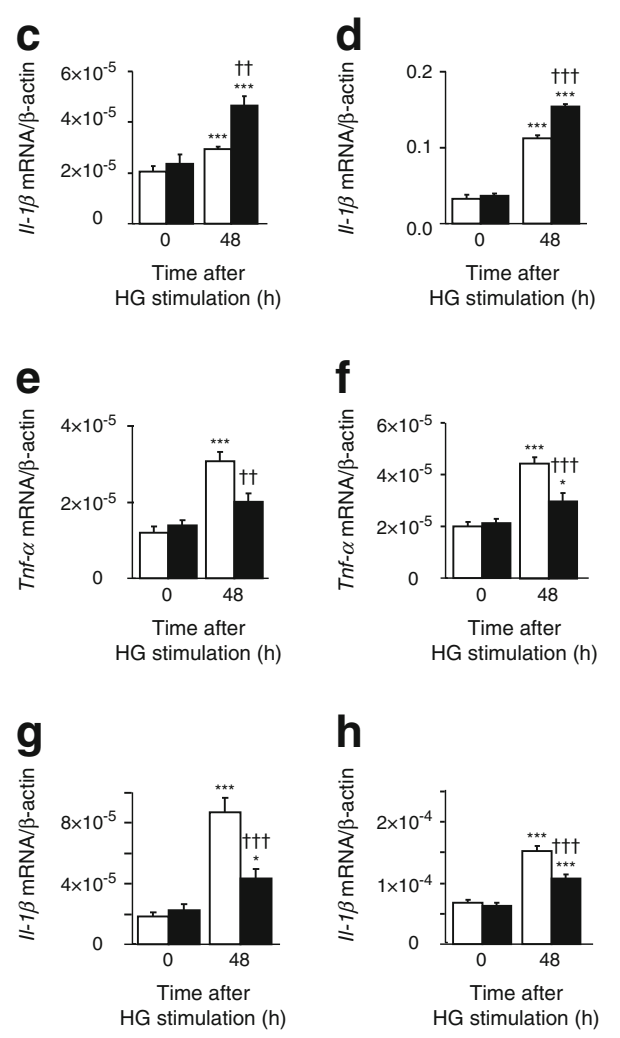

Fig. 6 Overexpression of miR-21 promotes, but inhibition of miR-21 reduces, high-glucose (HG)-induced abundance of proinflammatory markers in rat MCs and TECs. Abundance of Tnf- $\alpha$ in (a) MCs and (b) TECs with miR-21 overexpression; abundance of $I l-1 \beta$ in (c) MCs and (d) TECs with miR-21 overexpression; abundance of $\operatorname{Tnf}-\alpha$ in (e) MCs and (f) TECs with knockdown of miR-21; and abundance of $I l-1 \beta$ in (g) MCs and (h) TECs with knockdown of miR-21. miR-21 overexpression further promoted abundance of pro-inflammatory markers in MCs and TECs under diabetic conditions. Knockdown of miR-21 in MCs and TECs attenuates levels of proinflammatory markers induced by high glucose. Each bar represents the mean \pm SEM for at least three independent experiments. (a-d) White bars, + control vectors (CV); black bars, + miR-21 overexpression plasmids. (e-h) White bars, + CV; black bars, + miR-21 KD plasmids. ${ }^{*} p<0.05,{ }^{* *} p<0.01,{ }^{* * *} p<$ 0.001 compared with time $0 ;{ }^{\dagger \dagger} p<0.01,{ }^{\dagger \dagger} p<0.001$ compared with $\mathrm{CV}$ at the same time point

TGF- $\beta$ and NF- $\mathrm{KB}$ pathways by blocking the activation of Smad 3 and stimulating $\operatorname{I} \kappa \mathrm{B} \alpha$ production, respectively $[16$, 23]. Now, we demonstrate that knockdown of miR-21 in diabetic kidneys restores Smad7 levels and reduces phosphorylation of Smad3 and NF-kB/p65. Results from in vitro studies confirm the ability of miR-21 to reduce Smad7 content by binding to the Smad7 3'-UTR. As Smad7 knockout mice develop more severe renal fibrosis in both obstructive nephropathy and diabetic kidney disease as renal TGF- $\beta$ / Smad3 signalling is increased [24, 27], we also demonstrated that knockdown of Smad7 in kidney cells increases renal fibrosis and inflammation, as observed when miR-21 was overexpressed. More importantly, we showed that the renal fibrosis and inflammation present under diabetic conditions associated with miR-21 overexpression were reversed by restoration of target protein, Smad7, levels when transfection of miR-21-expressing cells with the Smad7 expression construct rendered miR-21 insensitive by deletion of its 3'-UTR. Thus the possible mechanism by which miR-21 regulates high-glucose-induced renal fibrosis and inflammation has been deduced to be via targeting of Smad7 so as to induce the TGF- $\beta$ and NF- $\mathrm{KB}$ signalling pathways. MiR-21 may function in a feed-forward loop, which amplifies TGF- $\beta$ signalling during renal injury.

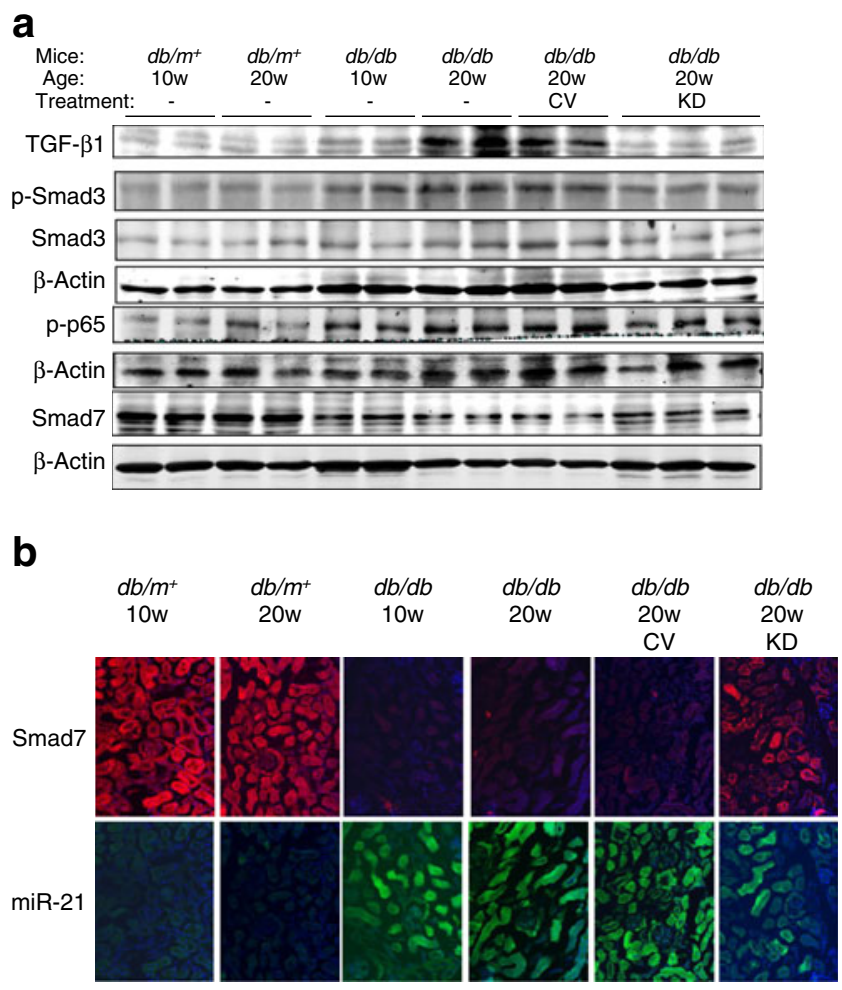

Fig. 7 Knockdown of renal miR-21 blocks activation of the TGF- $\beta$ / Smad and NF-kB signalling pathways by targeting Smad7 production. (a) Representative western blot analyses of renal TNF- $\beta 1$, Smad7, total Smad3 (Smad3), phosphorylated Smad3 (P-Smad3) and NF-kB/ p65 (P-p65) in $d b / d b$ mice. (b) Combination of immunohistochemical staining with Smad7 antibody (red) and in situ hybridisation with FITC-miR-21 (green). Note that knockdown of miR-21 activates the TGF- $\beta /$ Smad 3 and NF- $\mathrm{kB} / \mathrm{p} 65$ signalling pathways by reducing renal Smad7 abundance. In $d b / d b$ mice at age 20 weeks, expression levels of miR-21 are raised and endogenous Smad7 abundance is reduced. Knocking down miR-21 restores Smad7 levels in diabetic kidneys. Each bar represents the mean \pm SEM for at least eight mice. Magnification: $\times 400$. CV, control vectors; KD, miR-21 KD plasmids; w, weeks 
Fig. 8 Overexpression of Smad7 suppresses miR-21-promoted fibrosis and inflammation, and Smad7 is a target of miR-21 during renal fibrosis and inflammation. Real-time PCR results of (a) Smad7, (b) collagen I and (c) Tnf- $\alpha$ in MCs with Dox-induced overexpression of miR-21. Real-time PCR results of (d) Smad7, (e) collagen I and (f) Tnf- $\alpha$ in TECs with Dox-induced overexpression of miR-21. (g) Representative western blots of Smad7 and collagen I when Smad7 is overexpressed in Dox-induced miR-21-overexpressing TECs. (h) Real-time PCR analyses of Tnf- $\alpha$ when Smad7 is overexpressed in Dox-induced miR-21overexpressing TECs. (i) miR-21 inhibition of luciferase reporter activity in vectors with different segments of Smad7 3'-UTR. Transient transfection with Smad7 siRNA in (a) MCs and (b) TECs suppresses HG-induced levels of collagen I and TNF- $\alpha$. In contrast, overexpression of Smad7 in TECs suppresses miR-21-promoted production of collagen I and TNF- $\alpha$ in a dose-dependent manner (a-f). Furthermore, overexpression of miR-21 inhibits luciferase reporter activity in vectors with the sequences of the Smad7 3'-UTR containing an miR-21-binding site (i). No change in luciferase activity is seen in plasmids with mutated miR-21binding site or sequence without miR-21-binding site. Each bar represents the mean \pm SEM for at least three independent experiments. ${ }^{*} p<0.05$, ${ }^{* *} p<0.01, * * * p<0.001$ compared with time $0 ;{ }^{\dagger} p<0.05,{ }^{\dagger \dagger} p<0.01$, ${ }^{\dagger \dagger} p<0.001$ as indicated or compared with scrambled siRNA (Scr siRNA) or CV at the same time point, or Dox $(-)$. (a-f, h) White bars $0 \mathrm{~h}$; black bars, $48 \mathrm{~h}$. (i) White bars, + CV; black bars, +OE. CV, control vectors; $\mathrm{OE}$, miR-21 overexpression plasmids. Dox-induced miR-21-overexpressing TECs were transfected with 1,2 and $4 \mu \mathrm{g} /$ well of pcDNA3.1-Smad7 expression plasmid

However, the precise mechanism by which miR-21 affects fibrosis and inflammation may also be related to other putative target genes of miR-21. Studies of cardiac fibrosis indicate that SPRY and PTEN are potential targets of miR-21 [26, 28]. Recent studies of miR-21 knockout mice show that miR-21 promotes renal fibrosis by silencing metabolic pathways via suppression of peroxisome proliferatoractivated receptor- $\alpha$ [11]. Another study on diabetic kidney injury has shown that PRAS40, a negative regulator of TORC1, is a target of miR-21 [25]. For renal inflammation, we and others have shown that blocking miR-21 reduces macrophage infiltration in diseased kidneys [12]. However, other studies demonstrate anti-inflammatory properties of miR-21 in macrophages by targeting the proinflammatory programmed cell death 4 gene (PDCD4) [29, 30]. Negative correlation between miR-21 and PDCD4 has been reported in TECs, with induction of ischaemia [31]. Further studies should be performed to clarify whether miR-21 regulates inflammation in a cell-type-dependent fashion.

Although our present findings are consistent with previous studies showing that miR-21 plays a pathological role in tumorigenesis [32, 33], immunity [34, 35], inflammation [36] and fibrosis [7, 12, 22, 26], they are inconsistent with a report on miR-21 during diabetic kidney injury suggesting that it has a protective role in DN [37]. In that report, Zhang et al showed by microarray analysis that renal miR-21 levels were downregulated threefold in 8 -week-old $d b / d b$ mice. In addition, overexpression of miR-2 1 by intraperitoneal injection in 4-week-old mice improved kidney function and inhibited glomerular expansion at 8 weeks of age. The
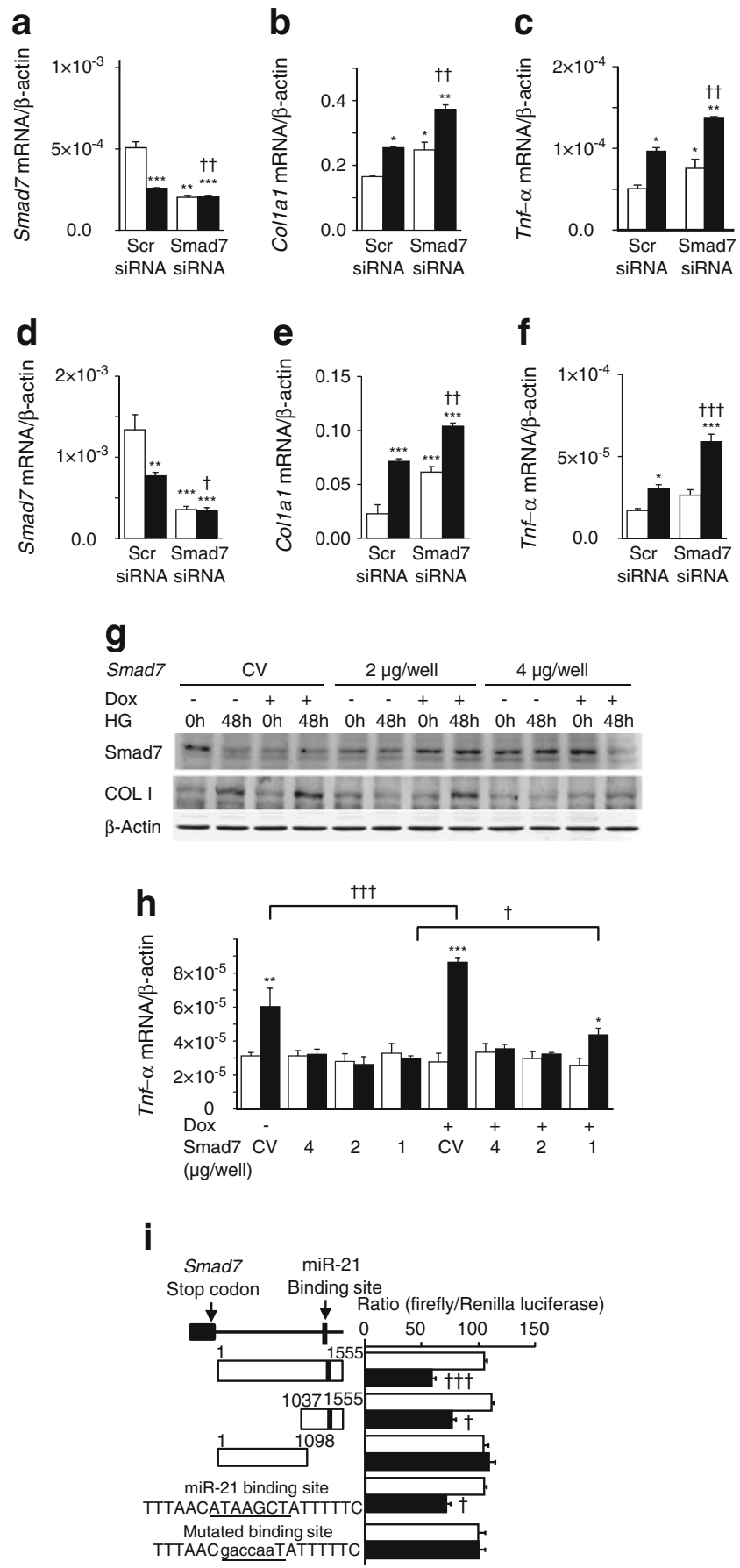

authors suggest that miR-21 suppresses Pten expression in MCs and diabetic kidneys, resulting in mesangial hypertrophy [37]. Although these discrepancies are as yet unexplained, the differences in disease nature, conditions and timing should be considered. First, we used BKS.Cg-Dock $7^{m}+/+L e p r^{d b} / \mathrm{J}$ with a C57BLKS/J background because it is widely used as a type 2 diabetic mouse model. Zhang et al used C57BL $/ 6 \mathrm{~J}^{\mathrm{Lep}}$ mice [37], and the diabetic phenotype of this strain has been reported to be less severe than that in C57BLKS/J [38]. Second, in the present study, we chose to study $d b / d b$ mice 
at the age of 10 weeks because $d b / d b$ mice rapidly develop hyperglycaemia between 6 and 10 weeks of age and diabetic renal injury occurs after 10 weeks of age [39-41]. In the study by Zhang et al [37], miR-21 treatment in mice started at the age of 4 weeks, before the increase in microalbuminuria. Third, we used ultrasound-microbubble-mediated gene transfer to deliver miR-21 KD plasmids specifically into the living kidneys [16]. However, Zhang et al delivered miR-21overexpression plasmids into diabetic kidneys by systemic intraperitoneal injection. However, the quantitative measurement of miR-21 abundance and functional role of miR-21 in kidneys and cells under diabetic conditions have not been investigated, which prevents further discussion about the discrepancies between these studies. In the present study, the pathological role of miR-21 during diabetic kidney injury is based on two observations. On the one hand, miR-21 levels were further increased in 20-week-old $d b / d b$ mice compared with 10-week-old mice, when hyperglycaemia and microalbuminuria worsen. On the other hand, suppression of miR-21 ameliorated progression of DN at the age of 20 weeks.

In summary, we propose that miR-21 is an important component of high-glucose-induced signalling, as upregulation of miR-21 reduces Smad7 abundance, increasing highglucose-induced fibrosis and NF-kB-mediated inflammation. Delivery of miR-21 KD plasmids offers an alternative therapeutic treatment for preventing diabetic kidney injury.

Acknowledgements We are grateful to F. A. Pereira of the Huffington Center on Aging, Baylor College of Medicine, Houston, TX, USA and D. J. Wilmshurst of the Research Administration Office, the Chinese University of Hong Kong, for commenting on a draft of this paper.

Funding This work was supported by grants from the Research Grant Council of Hong Kong (RGC GRF 468711, 469110, 768409 and CUHK5/CRF/09 to H.Y. Lan; GRF 464010, 463612, 763908, and 764109 to A.C.K. Chung) and the Focused Investment Scheme B (1902061) and by direct grants from the Chinese University of Hong Kong (2041656 and 2041735 to A.C.K. Chung), 2011 Research Grant from Hong Kong Society of Nephrology (6903213 to A.C.K. Chung), National Natural Science Foundation of China (General Program, 81170681 to A.C.K. Chung), and Major State Basic Research Development Program of China (973 program, No.2012CB517700 to H.Y. Lan).

Duality of interest The authors declare that there is no duality of interest associated with this manuscript.

Contribution statement XZ, YD, HC, XMM, RL, WY and ACKC designed the study, performed the research and analysed the data. HYL and FFH analysed data and revised the manuscript. ACKC was responsible for the conception of the study and drafting the article. All authors contributed to writing the paper and approved the final version.

\section{References}

1. Kanwar YS, Wada J, Sun L et al (2008) Diabetic nephropathy: mechanisms of renal disease progression. Exp Biol Med (Maywood) 233:4-11
2. Soldatos G, Cooper ME (2008) Diabetic nephropathy: important pathophysiologic mechanisms. Diabetes Res Clin Pract 82(Suppl 1):S75-S79

3. Ziyadeh FN (2004) Mediators of diabetic renal disease: the case for Tgf-Beta as the major mediator. J Am Soc Nephrol 15(Suppl 1):S55-S57

4. Yaswen L, Kulkarni AB, Fredrickson T et al (1996) Autoimmune manifestations in the transforming growth factor-beta 1 knockout mouse. Blood 87:1439-1445

5. Kantharidis P, Wang B, Carew RM, Lan HY (2011) Diabetes complications: the microRNA perspective. Diabetes 60:18321837

6. Lan HY (2011) Diverse roles of TGF-beta/Smads in renal fibrosis and inflammation. Int J Biol Sci 7:1056-1067

7. Zhong X, Chung AC, Chen HY, Meng XM, Lan HY (2011) Smad3-mediated upregulation of miR-21 promotes renal fibrosis. Clin J Am Soc Nephrol 22:1668-1681

8. Chung AC, Huang XR, Meng X, Lan HY (2010) miR-192 mediates TGF-beta/Smad3-driven renal fibrosis. J Am Soc Nephrol 21:1317-1325

9. Qin W, Chung AC, Huang XR et al (2011) TGF-\{beta $\} /$ Smad3 signaling promotes renal fibrosis by inhibiting miR-29. Clin J Am Soc Nephrol 22:1462-1474

10. Noetel A, Kwiecinski M, Elfimova N, Huang J, Odenthal M (2012) microRNA are central players in anti- and profibrotic gene regulation during liver fibrosis. Front Physiol 3:49

11. Chau BN, Xin C, Hartner J et al (2012) MicroRNA-21 promotes fibrosis of the kidney by silencing metabolic pathways. Sci Transl Med 4:121ra118

12. Zarjou A, Yang S, Abraham E, Agarwal A, Liu G (2011) Identification of a microRNA signature in renal fibrosis: role of miR-21. Am J Physiol Ren Physiol 301:F793-F801

13. Li JH, Huang XR, Zhu HJ, Johnson R, Lan HY (2003) Role of TGF-beta signaling in extracellular matrix production under high glucose conditions. Kidney Int 63:2010-2019

14. Chung AC, Zhang H, Kong YZ et al (2010) Advanced glycation end-products induce tubular CTGF via TGF-beta-independent Smad3 signaling. J Am Soc Nephrol 21:249-260

15. Hou CC, Wang W, Huang XR et al (2005) Ultrasound-microbubblemediated gene transfer of inducible Smad7 blocks transforming growth factor-beta signaling and fibrosis in rat remnant kidney. Am J Pathol 166:761-771

16. Lan HY, Mu W, Tomita $\mathrm{N}$ et al (2003) Inhibition of renal fibrosis by gene transfer of inducible Smad7 using ultrasound-microbubble system in rat UUO model. J Am Soc Nephrol 14:1535-1548

17. Ng YY, Hou CC, Wang W, Huang XR, Lan HY (2005) Blockade of NFkappaB activation and renal inflammation by ultrasoundmediated gene transfer of Smad7 in rat remnant kidney. Kidney Int Suppl: S83-91

18. Ka SM, Huang XR, Lan HY et al (2007) Smad7 gene therapy ameliorates an autoimmune crescentic glomerulonephritis in mice. J Am Soc Nephrol 18:1777-1788

19. Kloosterman WP, Wienholds E, de Bruijn E, Kauppinen S, Plasterk RH (2006) In situ detection of miRNAs in animal embryos using LNA-modified oligonucleotide probes. Nat Methods 3:27-29

20. Williams MD, Nadler JL (2007) Inflammatory mechanisms of diabetic complications. Curr Diab Rep 7:242-248

21. Navarro-Gonzalez JF, Mora-Fernandez C (2008) The role of inflammatory cytokines in diabetic nephropathy. Clin J Am Soc Nephrol 19:433-442

22. Liu G, Friggeri A, Yang Y et al (2010) miR-21 mediates fibrogenic activation of pulmonary fibroblasts and lung fibrosis. J Exp Med 207:1589-1597

23. Wang W, Huang XR, Li AG et al (2005) Signaling mechanism of TGF-beta1 in prevention of renal inflammation: role of Smad7. J Am Soc Nephrol 16:1371-1383 
24. Chen H, Huang XR, Wang W et al (2010) The protective role of Smad7 in diabetic kidney disease: mechanism and therapeutic potential. Diabetes 60:590-601

25. Dey N, Das F, Mariappan MM et al (2011) MicroRNA-21 orchestrates high glucose-induced signals to TOR complex 1 , resulting in renal cell pathology in diabetes. J Biol Chem 286:25586-25603

26. Thum T, Gross C, Fiedler J et al (2008) MicroRNA-21 contributes to myocardial disease by stimulating MAP kinase signalling in fibroblasts. Nature 456:980-984

27. Chung AC, Huang XR, Zhou L, Heuchel R, Lai KN, Lan HY (2009) Disruption of the Smad7 gene promotes renal fibrosis and inflammation in unilateral ureteral obstruction (UUO) in mice. Nephrol Dial Transplant 24:1443-1454

28. Roy S, Khanna S, Hussain SR et al (2009) MicroRNA expression in response to murine myocardial infarction: miR-21 regulates fibroblast metalloprotease- 2 via phosphatase and tensin homologue. Cardiovasc Res 82:21-29

29. Sheedy FJ, Palsson-McDermott E, Hennessy EJ et al (2010) Negative regulation of TLR4 via targeting of the proinflammatory tumor suppressor PDCD4 by the microRNA miR-21. Nat Immunol 11:141-147

30. Merline R, Moreth K, Beckmann J et al (2011) Signaling by the matrix proteoglycan decorin controls inflammation and cancer through PDCD4 and MicroRNA-21. Sci Signal 4:ra75

31. Godwin JG, Ge X, Stephan K, Jurisch A, Tullius SG, Iacomini J (2010) Identification of a microRNA signature of renal ischemia reperfusion injury. Proc Natl Acad Sci U S A 107:14339-14344

32. Fu X, Han Y, Wu Y et al (2011) Prognostic role of microRNA-21 in various carcinomas: a systematic review and meta-analysis. Eur J Clin Invest 41:1245-1253
33. Pan X, Wang ZX, Wang R (2011) MicroRNA-21: a novel therapeutic target in human cancer. Cancer Biol Ther 10: $1224-1232$

34. Liu PT, Wheelwright M, Teles R et al (2012) MicroRNA-21 targets the vitamin D-dependent antimicrobial pathway in leprosy. Nat Med 18:267-273

35. Lu TX, Hartner J, Lim EJ et al (2011) MicroRNA-21 limits in vivo immune response-mediated activation of the IL-12/IFN-gamma pathway, Th1 polarization, and the severity of delayed-type hypersensitivity. J Immunol 187:3362-3373

36. Roggli E, Britan A, Gattesco S et al (2010) Involvement of microRNAs in the cytotoxic effects exerted by proinflammatory cytokines on pancreatic beta-cells. Diabetes 59:978-986

37. Zhang Z, Peng H, Chen J et al (2009) MicroRNA-21 protects from mesangial cell proliferation induced by diabetic nephropathy in $\mathrm{db} /$ db mice. FEBS Lett 583:2009-2014

38. Breyer MD, Bottinger E, Brosius FC 3rd et al (2005) Mouse models of diabetic nephropathy. J Am Soc Nephrol 16:27-45

39. Cohen MP, Clements RS, Cohen JA, Shearman CW (1996) Prevention of decline in renal function in the diabetic $\mathrm{db} / \mathrm{db}$ mouse. Diabetologia 39:270-274

40. Lim AK, Ma FY, Nikolic-Paterson DJ, Thomas MC, Hurst LA, Tesch GH (2009) Antibody blockade of c-fms suppresses the progression of inflammation and injury in early diabetic nephropathy in obese $\mathrm{db} / \mathrm{db}$ mice. Diabetologia 52:1669-1679

41. Arakawa K, Ishihara T, Oku A et al (2001) Improved diabetic syndrome in $\mathrm{C} 57 \mathrm{BL} / \mathrm{KsJ}-\mathrm{db} / \mathrm{db}$ mice by oral administration of the $\mathrm{Na}(+)$-glucose cotransporter inhibitor T-1095. Br J Pharmacol 132:578-586 